\title{
ROLE OF CHEWING GUM ON EARLY RECOVERY OF FEMALES AFTER CAESAREAN SECTION
}

\author{
Rabia Wajid, ${ }^{1}$ Ghazala Huma, ${ }^{2}$ Iram Mobusher $^{3}$
}

\begin{abstract}
Background: The incidence of Caesarean section is increasing day by day, which is usually conducted under spinal anesthesia. It is done on lower abdominal region and for this reason; the working of digestive system can slow down in the coming period. This is known as ileus, and is distressing for the patient but the way to prevent this problem, is there. One possible way of avoiding this consequence is chewing gum which tricks the body into thinking it is eating making the bowel start its function again.
\end{abstract}

Objective: To compare the effect of chewing gum as compared to no intervention for early recovery of bowel system after lower segment caesarean section at term.

Methodology: This randomized controlled trial was conducted on 100 females who were planned to undergo caesarean section. Informed consent was obtained and demographic details were noted. In group A,

Wajid R. ${ }^{1}$

Senior Registrar, Department of Obstetrics \& Gynaecology Lady Willingdon Hospital, Lahore

Huma G. ${ }^{2}$

Women Medical Officer, Department of Obstetrics \& Gynaecology, Lady Willingdon Hospital, Lahore

Mobusher I. ${ }^{3}$

Senior Registrar, Department of Obstetrics \& Gynaecology Lady Willingdon Hospital, Lahore females were suggested to take chewing gum after 6 hours of surgery to chew for 60 minutes while in group B, females were not subjected to chewing gum. They were followed-up in wards and time required for first bowel sound and first fecal discharge was noted and compared in both groups by using t-test.

Results: The mean age of females was $24.48 \pm 1.99$ years. The females presented at mean gestational age of $38.76 \pm 0.87$ weeks. The mean BMI of females was $25.49 \pm 2.29 \mathrm{~kg} / \mathrm{m}^{2}$. Total duration of surgery required for c-section including anesthesia time was $42.28 \pm$ 2.38 minutes. After surgery, the mean duration between c-section and first onset of feeling of hunger was $11.38 \pm 3.14$ hours in chewing gum group and $16.84 \pm$ 0.49 hours in control group. The mean duration between c-section and first bowel sound was $21.39 \pm 0.68$ hours in chewing gum group and in control group were $28.27 \pm 0.60$ hours. The mean duration between csection and first passage of flatus was $25.94 \pm 0.71$ hours in chewing gum group and $32.00 \pm 0.77$ hours in control group. The mean duration between c-section and first defecation was $31.56 \pm 0.81$ hours in chewing gum group and $41.28 \pm 0.80$ hours in control group. The difference was significant and chewing gum group required less duration for feel of hunger, first bowel sound, passage of flatus and defecation than control group $(\mathrm{p}<0.05)$.

Conclusion: In conclusion, use of chewing is effective and beneficial in early recovery of females after caesarean section in terms of early return of bowel activities. 
Key words: Bowel Sound, Caesarean Section, Chewing Gum, Digestive System, Fecal Discharge, Ileus.

\section{Introduction}

Caesarean section is one of the most common obstetrical surgery carried out all over the world. ${ }^{1}$ The incidence of caesarean sections has shown drastic increase each year worldwide. Consequently, postoperative care of such females required some attention. ${ }^{2}$ Caesarean section is associated with central nervous system (CNS)changes in postoperative period, leading to decreased bowel movements and associated problems among women. ${ }^{3}$

Gastrointestinal complications are common after caesarean section. Many relevant studies have reported different results about the impact of early feeding after caesarean section and its relationship to various parameters like vomiting, nausea, flatulence and ileus. ${ }^{4}$ Postoperative ileus (POI) usually follows caesarean section. ${ }^{1}$

Postoperative ileus is illustrated by a temporary inhibition of gut motility leading to wide range of events culminating in increased burden on healthcare. ${ }^{5,6}$ Postoperative ileus is defined as temporary inhibition of coordinated bowel function after surgery, and may follow surgery involving abdominal viscera. It is one of the reasons for prolonged hospital stay and increased demand from healthcare resources. ${ }^{7,8}$ There are many causes for POI; therefore, identification and rectification of these factors is required. These measures include thorough preoperative assessment; avoidance of undue gut handling; early feeding; avoidance of unnecessary intervention; regional analgesia; and gum chewing. ${ }^{9-11}$

Since, gynecological surgeons have been traditionally allowing oral intake to caesarean patients post operatively after they have initiated their bowel function diagnosed by appearance of gut sounds, passage of stool and feeling of hunger. When the patient passes stool or flatus it indicates a return of bowel motility. Research has shown that early start of feeding after caesarean section before passing stool or flatus, such a gap in the start of feeding culminates in increased tissue damage, compromised healing of wound, increased chances of infection leading to increased requirement of parenteral support. It becomes a burden on the hospital and the family. $3,13,14$
Therefore, several remedies have come up to quicken the return of gut motility after caesarean section, for example starting oral fluid intake, mobilization and gum chewing. ${ }^{15-17}$ Several studies have been conducted on gum chewing but contraindications are present regarding the effectiveness of gum chewing on early bowel movements. ${ }^{18}$ So we conducted this study to find the beneficiary role of gum chewing in early bowel motility of post caesarean patients.

\section{Patients and Method}

This single blind randomized controlled trial study was carried out on 100 pregnant women candidates for caesarean section with spinal anesthesia in Department of Obstetrics \& Gynecology, Lady Willingdon Hospital, Lahore during January 2015 to March 2015. Written informed consent was obtained all women. All enrolled women were allocated using a computer-generated random sequence from a statistics program. All caesarean section were carried out by the same Obstetrician. A transverse incision on the uterus and a Pfannenstiel incision on the abdomen were performed.

Demographic information on all variables women included; patient's age, gestational age, body mass index, number of miscarriages, type of caesarean (elective or emergency) and duration of surgery. Exclusion criteria were: women consuming drugs, especially opioids, water and electrolyte imbalance, inflammation of pancreas and peritoneum, intestinal surgery (hernioplasty) in the past, diabetes, pre-eclampsia, prolonged rupture of membranes, hypothyroidism, and muscular and neurological disorders. The women in the chewing gum group chewed sugar-free gum for at least one hour, three times daily from six hours after surgery until being discharged. Commercially available sugarfree gum was prescribed. The oral intake of clear fluids and soft foods was initiated on first postoperative day. Around 48 hours following operation, the women were discharged when they had stable vital signs, ability to ambulate and urinate independently, defecate and ability to tolerate solid food. All women were vigilantly seen till discharge from hospital, and the times to the first bowel sounds, normal intestinal sounds, and the time to the first defecation were noted down. All data analysis was performed with IBM SPSS (version 21.0). The data were analyzed by t test and chi square, depending on the nature of variable. A $p$-value $\leq 0.05$ was considered statistically significant. 


\section{Results}

In this trial, the mean age of females in chewing gum group was $23.72 \pm 1.37$ years while the mean age of females in control group was $25.24 \pm 2.24$ years. The females presented at mean gestational age of $38.90 \pm$ 0.75 weeks in chewing gum group, while at $38.62 \pm$ 0.97 weeks in control group. In both study groups, there were $13(52 \%)$ females who were booked for delivery with our hospital while 12 (48\% in each group) were non-booked and presented at the time of delivery (see table 1 ).

There were $24(48 \%)$ females who did not have any history of miscarriage. However, 14 (28\%) females reported that they had 1 miscarriage, $11(22 \%)$ had history of 2 miscarriages while only $1(2 \%)$ female had history of 3 miscarriages (see fig 1). The mean BMI of females in chewing gum group was $25.39 \pm 1.10$ $\mathrm{kg} / \mathrm{m}^{2}$ and the mean BMI of females in control group was $25.58 \pm 3.08 \mathrm{~kg} / \mathrm{m}^{2}$. Total duration of surgery required for c-section including anesthesia time was $42.72 \pm 1.97$ minutes in chewing gum group and 41.84 \pm 2.70 minutes in control group (see table 1 ).

After surgery, the mean duration between c-section and first onset of hunger was $11.38 \pm 3.14$ hours in chewing gum group and $16.84 \pm 0.49$ hours in control group. The difference was significant and chewing gum group required less duration than control group

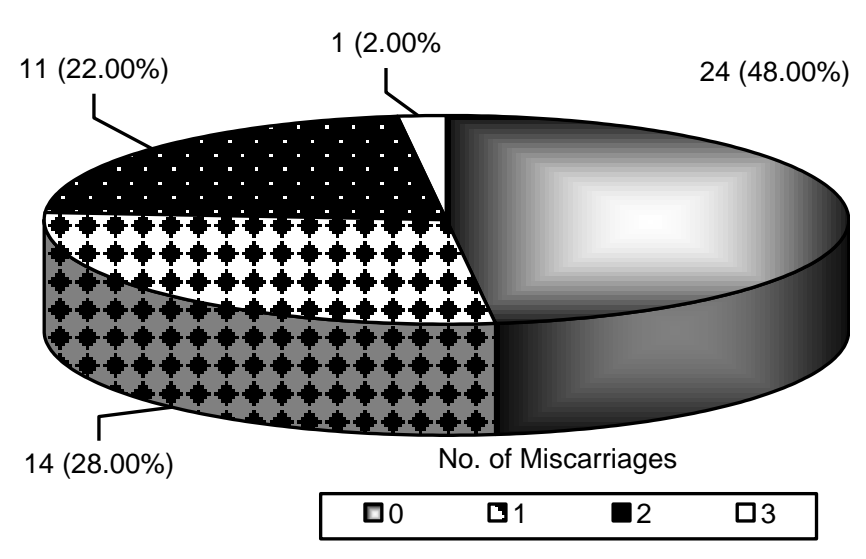

Fig. 1: Distribution of history of miscarriages reported by females.

$(\mathrm{p}<0.05)$. The mean duration between $\mathrm{c}$-section and first bowel sound was $21.39 \pm 0.68$ hours in chewing gum group which was significantly less than control group which required mean duration of $28.27 \pm 0.60$ hours for first bowel sound after c-section. Similarly, the mean duration between c-section and first passage of flatus was $25.94 \pm 0.71$ hours in chewing gum group and $32.00 \pm 0.77$ hours in control group. The difference was significant and chewing gum group required less duration than control group $(\mathrm{p}<0.05)$. The mean

Table 1: Baseline characteristics of patients included in the study.

\begin{tabular}{|l|l|l|l|}
\hline \multicolumn{1}{|c|}{ Groups } & Chewing Gum & \multicolumn{1}{c|}{ Control } & \multicolumn{1}{c|}{ Overall } \\
\hline $\mathrm{N}$ & 25 & 25 & 50 \\
\hline Age (years) & $23.72 \pm 1.37$ & $25.24 \pm 2.24$ & $24.48 \pm 1.99$ \\
\hline Gestational age (weeks) & $38.90 \pm 0.75$ & $38.62 \pm 0.97$ & $38.76 \pm 0.87$ \\
\hline Booking status (Booked / Unbooked) & $13 / 12$ & $13 / 12$ & $26 / 24$ \\
\hline BMI $\left(\mathrm{kg} / \mathrm{m}^{2}\right.$ ) & $25.39 \pm 1.10$ & $25.58 \pm 3.08$ & $25.49 \pm 2.29$ \\
\hline Duration of Surgery (min) & $42.72 \pm 1.97$ & $41.84 \pm 2.70$ & $42.28 \pm 2.38$ \\
\hline
\end{tabular}

Table 2: Outcome measures between both study groups after C-section.

\begin{tabular}{|l|c|c|c|}
\hline \multicolumn{1}{|c|}{ Groups } & Chewing Gum & Control & p-value \\
\hline Duration between surgery to First bowel sound (hours) & $21.39 \pm 0.68$ & $28.27 \pm 0.60$ & 0.000 \\
\hline Duration between surgery to first passage of flatus (hours) & $25.94 \pm 0.71$ & $32.00 \pm 0.77$ & 0.000 \\
\hline Duration between surgery to defecation (hours) & $31.56 \pm 0.81$ & $41.28 \pm 0.80$ & 0.000 \\
\hline Duration between surgery to first onset of hunger (hours) & $11.38 \pm 3.14$ & $16.84 \pm 0.49$ & 0.000 \\
\hline
\end{tabular}


duration between c-section and first defecation was $31.56 \pm 0.81$ hours in chewing gum group and $41.28 \pm$ 0.80 hours in control group. The difference was significant and chewing gum group required less duration than control group $(\mathrm{p}<0.05)$.

\section{Discussion}

This study was conducted on 100 pregnant females who were planned to undergo caesarean section at term. The mean age of females was $24.48 \pm 1.99$ years and they were present for delivery at gestational age of $38.76 \pm 0.87$ weeks. In our trial we included females with normal BMI $\left(25.49 \pm 2.29 \mathrm{~kg} / \mathrm{m}^{2}\right)$ so that BMI may not cause any confounding effect as very lean and obese female have some health issues which cause delay in wound healing and early recovery. In our trial the total duration of surgery required for c-section including anesthesia time was $42.28 \pm 2.38$ minutes.

In our trial, the mean duration between c-section and first onset of hunger was shorter in chewing group as compared to control group (11.38 \pm 3.14 hours versus $16.84 \pm 0.49$ hours, respectively, $\mathrm{p}<0.05)$. In our trial with chewing as compared to control group (i.e. $21.39 \pm 0.68$ hours versus $28.27 \pm 0.60$ hours, respectively, $\mathrm{p}<0.05)$. Similarly, the mean duration between c-section and first passage of flatus was significantly shorter with chewing as compared to control group (i.e. $25.94 \pm 0.71$ hours versus $32.00 \pm 0.77$ hours, respectively, $\mathrm{p}<0.05$ ). And the mean duration between c-section and first defecation was significantly shorter with chewing as compared to control group (i.e. 31.56 \pm 0.81 hours versus $41.28 \pm 0.80$ hours, respectively, $\mathrm{p}<0.05)$.

These evidences attained in our trial were supported by another trial conducted by Ledari et al., in 2012 and they reported that the mean time to first bowel sounds (21.1 versus 26.1 hours, $\mathrm{p}=0.016$ ), the first feeling of hunger (11.8 versus 14.5 hours, $p=0.050$ ), the first passage of flatus (24.8 versus 30.0 hours, $\mathrm{P}=0.002)$, the first defecation (30.6 versus 38.4 hours, $\mathrm{P}=0.0001)$ was much less compared to the control group. ${ }^{19}$ Afterwards Ledari et al, carried out one more trial and found that the functioning of the bowel started much earlier in the chewing gum group. ${ }^{18}$

The results of this study show a similarity with a study by Dehcheshmeh reporting $7.4 \pm 1.71$ hours and $15.7 \pm 3.44$ hours as the mean time to the first bowel movement in the gum and the control group respectively, ${ }^{7,20,21}$ the finding is also in consistent with Schu- ster study on the impact of gum chewing after sigmoid - colostomy surgery, in which the mean time interval to the first bowel movement was more in $\mathrm{s}$ the control group. Both groups showed a difference in the parameter of hunger but it did not have a statistical significance in the larger context. ${ }^{22}$

Nimarta et al, through a randomized trial observed that the mean time of return of bowel sounds after surgery was significantly lesser $(21.4 \pm 2.8 \mathrm{hr})$ in the experimental group than in the control group (23.7 \pm $2.8 \mathrm{hr}$ ). Similarly the mean time of passage of flatus after surgery was significantly lesser in $(58.2 \pm 9.3 \mathrm{hr})$ in the experimental group than in the control group $(65.6 \pm 6.4 \mathrm{hr})$. The mean time of return of appetite after surgery was significantly lesser $(59.9 \pm 9.8 \mathrm{hr})$ in experimental group than in the control group (67.2 \pm $7.6 \mathrm{hr}) .^{23}$

But the study by Akhlaghi et al, brought up controversy in term of these results. They found that both groups were dissimilar in terms of the feeling of bowel movement, but the mean difference between the two groups was not statistically significant $(14.7 \pm 6.5$ horses $16.6 \pm \mathrm{hrs})^{8}$

In a meta-analysis of six RCTs which included 939 women, presented pooled data demonstrating that gum chewing has shown better results to no gum chewing in terms of mean reduction of all the parameters under consideration in this study. No inference has come up for any harmful effects caused by this practice. An opinion was drawn through this meta-analysis that gum chewing has a beneficial role in bowel functioning. This safe and low cost intervention should be inculcated as a part of routine protocol postoperatively. $^{24}$

Another meta-analysis is of the opinion that chewing gum for $30-60$ minutes thrice daily is helpful in reduction of post operative ileus. A previous metaanalysis comprising of seventeen randomized controlled trials showed similar results in terms of parameters evaluated in the previous and index studies ${ }^{25,26}$

\section{Conclusion}

In conclusion, use of chewing is effective and beneficial in early recovery of females after caesarean section in terms of early return of bowel activities. Now in future we can recommend the use of chewing gum after c-section in our routine practice. This will help in early healing of wound as well as female will be able to move early after caesarean section. Further studies 
may be conducted on lower abdominal surgeries and effect of chewing gum use may be achieved. Moreover, large randomized controlled trials are required to be conducted on large sample sizes.

\section{References}

1. Mattei P, Rombesau JL. Review of the pathophysiology and management of postoperative ileus. World Journal of Surgery, 2006; 30 (8): 1382-91.

2. Fazel N, Tafazoli M, Ramezani M, Esmaili H. [The effect of superminet on post caesarean flatulence]. Res Sci J Ardabil Univ Med Sci Health Serv. 2005; 4 (14).

3. Hirayama I, Suzuki M, Ide M, Asao T, Kuwano H. Gum - chewing stimulates bowel motility after surgery for colorectal cancer. Hepato-gastroenterology, 2005; 53 (68): 206-8.

4. Adeli M, Razmjoo N, Tara F, Ebrahimzade S. Effect of Early Post Caesarean Feeding on Gastrointestinal Complications. Nursing and Midwifery Studies, 2013; 2 (2): 176.

5. Behm B, Stollman N. Postoperative ileus: etiologies and interventions. Clinical Gastroenterology and Hepatology, 2003; 1 (2): 71-80.

6. Doorly MG, Senagore AJ. Pathogenesis and clinical and economic consequences of postoperative ileus. Surgical Clinics of North America, 2012; 92 (2): 259-72.

7. Ghafouri A, Soroush A, Moeini N, Hedayat A, Khourgami Z. The efficacy of sugar free gum chewing after upper GI tract operation on ileus: a clinical trial. Iranian J Surg. 2008; 16 (1): 79-85.

8. Akhlaghi F, Pourjavad M, Mansouri A, Tara F, Vahedian M. Effect of Gum Chewing on Prevention of Post Caesarean Ileus. Hayat. 2009; 14 (2): 35-40.

9. Miedema BW, Johnson JO. Methods for decreasing postoperative gut dysmotility. The lancet oncology, 2003; 4 (6): 365-72.

10. Wind J, Hofland J, Preckel B, Hollmann MW, Bossuyt PM, Gouma DJ, et al. Perioperative strategy in colonic surgery; LAparoscopy and/or FAst track multimodal management versus standard care (LAFA trial). BMC surgery, 2006; 6 (1): 16.

11. Adanikin AI, Orji EO, Fasubaa OB, Onwudiegwu U, Ijarotimi OA, Olaniyan O. The effect of post-caesarean rectal misoprostol on intestinal motility. International Journal of Gynecology \& Obstetrics, 2012; 119 (2): 159-62.

12. Yaghmaei M, Arbabi Kalati F, Mokhtari M, Behzadian A. Comparison of oral intake profiles at 2 and 8 hours following caesarean section under spinal anesthesia. Zahedan Journal of Research in Medical Sciences, 2010; 11 (4): 0-.

13. Pearl ML, Frandina M, Mahler L, Valea FA, DiSilvestro PA, Chalas E. A randomized controlled trial of a regular diet as the first meal in gynecologic oncology patients undergoing intraabdominal surgery. Obstetrics \& Gynecology, 2002; 100 (2): 230-4.
14. Holte K, Kehlet H. Postoperative ileus: a preventable event. British Journal of surgery, 2000; 87 (11): 148093.

15. Izbizky G, Minig L, Sebastiani M, Otaño L. The effect of early versus delayed postcaesarean feeding on women's satisfaction: a randomised controlled trial. BJOG: An International Journal of Obstetrics \& Gynaecology, 2008; 115 (3): 332-8.

16. Kovavisarach E, Atthakorn M. Early versus delayed oral feeding after caesarean delivery. International Journal of Gynecology \& Obstetrics, 2005; 90 (1): 31-4.

17. Kafali H, Duvan CI, Gözdemir E, Simavli S, Onaran Y, Keskin E. Influence of gum chewing on postoperative bowel activity after caesarean section. Gynecologic and obstetric investigation, 2010; 69 (2): 84-7.

18. Ledari FM, Barat S, Delavar MA, Banihosini SZ, Khafri S. Chewing sugar-free gum reduces ileus after caesarean section in nulliparous women: a randomized clinical trial. Iranian Red Crescent Medical Journal, 2013; 15 (4): 330.

19. Ledari FM, Barat S, Delavar MA. Chewing gums has stimulatory effects on bowel function in patients undergoing caesarean section: A randomized controlled trial. Bosnian Journal of Basic Medical Sciences, 2012; 12 (4): 265 .

20. Dehcheshmeh FS. The effect of chewing sugar free gum after elective caesarean - delivery on return of bowel function in primiparous women. Qom University of Medical Sciences Journal, 2012; 4 (4).

21. Shang H, Yang Y, Tong X, Zhang L, Fang A, Hong L. Gum chewing slightly enhances early recovery from postoperative ileus after caesarean section: results of a prospective, randomized, controlled trial. American journal of perinatology, 2010; 27 (5): 387-91.

22. Schuster R, Grewal N, Greaney GC, Waxman K. Gum chewing reduces ileus after elective open sigmoid colectomy. Archives of Surgery, 2006; 141 (2): 174-6.

23. Nimarta N, Shruti RG. Effectiveness of chewing gum on bowel motility among the patients who have undergone abdominal surgery. Nurs Midwifery Res J. 2013; 9: 108-18.

24. Zhu YP, Wang WJ, Zhang SL, Dai B, Ye DW. Effects of gum chewing on postoperative bowel motility after caesarean section: a meta-analysis of randomised controlled trials. BJOG: An International Journal of Obstetrics \& Gynaecology, 2014; 121 (7): 787-92.

25. Li S, Liu Y, Peng Q, Xie L, Wang J, Qin X. Chewing gum reduces postoperative ileus following abdominal surgery: A meta-analysis of 17 randomized controlled trials. Journal of gastroenterology and hepatology, 2013; 28 (7): 1122-32.

26. Craciunas L, Sajid M, Ahmed AS. Chewing gum in preventing postoperative ileus in women undergoing caesarean section: a systematic review and metaanalysis of randomised controlled trials. BJOG: An International Journal of Obstetrics \& Gynaecology, 2014; 121 (7): 793-800. 